\title{
OPEN Circulating bile acids concentration is predictive of coronary artery disease in human
}

\author{
Caroline Chong Nguyen ${ }^{1,2,3,4,7}$, Denis Duboc ${ }^{2,7}$, Dominique Rainteau ${ }^{4}$, Harry Sokol ${ }^{4}$, \\ Lydie Humbert ${ }^{4}$, Philippe Seksik ${ }^{4}$, Adèle Bellino ${ }^{5}$, Hendy Abdoul ${ }^{5}$, Naïm Bouazza ${ }^{5}$, \\ Jean-Marc Treluyer ${ }^{5}$, Malika Saadi ${ }^{2}$, Karim Wahbi ${ }^{2}$, Heithem Soliman ${ }^{3}$, Benoit Coffin ${ }^{1,3}$, \\ André Bado ${ }^{1}$, Maude Le Gall ${ }^{1}$, Olivier Varenne ${ }^{2}$ \& Henri Duboc ${ }^{1,2,3,6 \bowtie}$
}

Synthetized by the liver and metabolized by the gut microbiota, BA are involved in metabolic liver diseases that are associated with cardiovascular disorders. Animal models of atheroma documented a powerful anti-atherosclerotic effect of bile acids (BA). This prospective study examined whether variations in circulating BA are predictive of coronary artery disease (CAD) in human. Consecutive patients undergoing coronary angiography were enrolled. Circulating and fecal BA were measured by high pressure liquid chromatography and tandem mass spectrometry. Of 406 screened patients, 80 were prospectively included and divided in two groups with $(n=45)$ and without $(n=35)$ CAD. The mean serum concentration of total BA was twice lower in patients with, versus without $C A D(P=0.005)$. Adjusted for gender and age, this decrease was an independent predictor of CAD. In a subgroup of 17 patients, statin therapy doubled the serum BA concentration. Decreased serum concentrations of BA were predictors of CAD in humans. A subgroup analysis showed a possible correction by statins. With respect to the anti-atherosclerotic effect of BA in animal models, and their role in human lipid metabolism, this study describe a new metabolic disturbance associated to CAD in human.

Cardiovascular diseases (CVD) remain a major cause of death, whose prevention of modifiable risk factors can lower the incidence of ischemic events ${ }^{1,2}$. Increased morbidity and mortality by CVD are undoubtedly observed during Non Alcoholic Fatty Liver Disease (NAFLD) ${ }^{3}$, supported by overlapping risk factors. Beyond the clinical association, fundamental research describes numerous mechanistic axes that are shared between CVD and NAFLD, including disorders of lipid-glucid metabolism, systemic and local inflammation, and fibrosis ${ }^{4,5}$. In all these themes, BA have been associated in the literature, either with a protective ${ }^{6-8}$ or a worsening $^{9-11}$ role in $^{-1}$ these processes.

Acting as hormones through the vascular system, BA are "wandering molecules" which regulate the metabolism and inflammation in metabolic disorders ${ }^{12}$ : the permanent loop between their secretion in the gut lumen, their microbiota transformation, their ileal reabsorption and their vascular transportation through the portal blood to the liver-known as the entero-hepatic cycle- is a bridge between the microbiota and the host. The synthesis by the liver from cholesterol under the primary cholic (CA) and chenodeoxycholic acid (CDCA) forms is the first metabolic step ${ }^{13}$. Once conjugated to taurin or glycin, they are stocked in bile. Upon postprandial emptying of the gallbladder, they reach the lumen and promote the micellization of alimentary fat. In the gut, they are metabolized by the microbiota, consisting of a bacterial deconjugation followed by a transformation (dehydroxylation), leading to the secondary deoxycholic acid (DCA) and lithocholic acid (LCA) ${ }^{13}$. By modifying the stereospatial configuration of BA, the microbiota directly influences the composition of the bile acids pool ${ }^{13}$, as well as their binding and activation properties to their specific receptors ${ }^{14,15}$. This loop is closed when $95 \%$ of the BA are reabsorbed in the ileum, returning to the liver via the portal circulation. In the peripheral blood,

${ }^{1}$ Centre de Recherche Sur I'inflammation, Inserm, UMR 1149, Université de Paris, 75018 Paris, France. ${ }^{2}$ Cardiology Department, Cochin Hospital, Assistance Publique-Hôpitaux de Paris, Université de Paris, 75014 Paris, France. ${ }^{3}$ Department of Hepato Gastro Enterology and University of Paris, Louis Mourier Hospital, APHP, 92700 Colombes, France. ${ }^{4}$ Inserm, Centre de Recherche Saint-Antoine, CRSA, AP-HP, Hôpital Saint Antoine, Service de Gastroentérologie, Sorbonne Université, 75012 Paris, France. ${ }^{5}$ Unite de Recherche Clinique-Centre Dinvestigation Clinique Necker/Cochin, Hôpital Tarnier, Université de Paris, 75006 Paris, France. ${ }^{6}$ INSERM UMRS 1149, Université de Paris, 16 rue Henri Huchard, 75890 Paris Cedex 18, France. ${ }^{7}$ These authors contributed equally: Caroline Chong Nguyen and Denis Duboc ${ }^{\square}$ email: henri.duboc@aphp.fr 


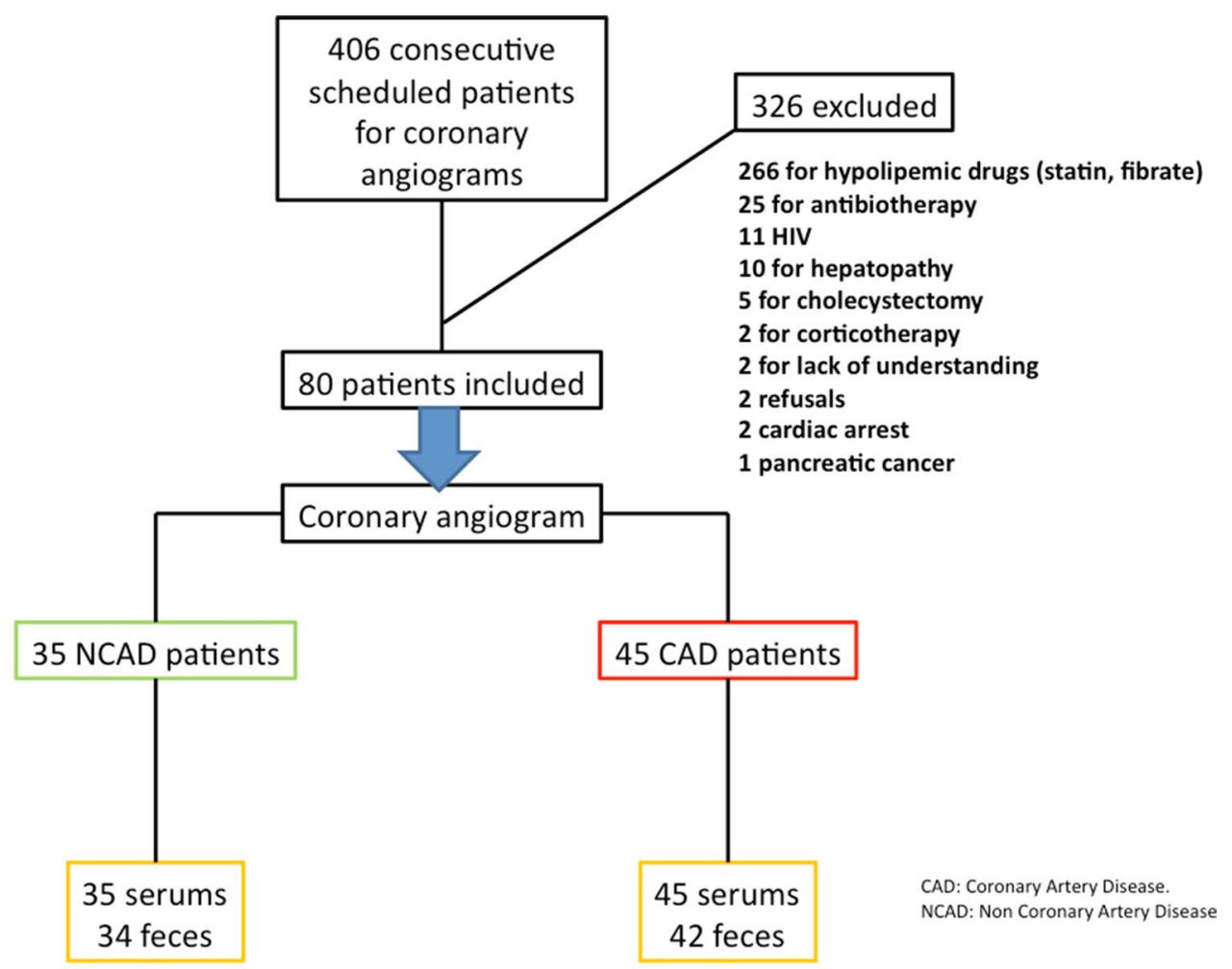

Figure 1. Flow chart of patients screened, excluded and enrolled in each study group, and their contributions of serum and fecal samples.

BA circulate instable concentrations, with a prominent postprandial peak (the "spill over") following their rapid reabsorption by the gut $^{16}$.

The two main BA receptors are the membrane TGR $5^{17}$ and the nuclear FXR ${ }^{18}$. Both are implicated in NAFLD and in CAD pathogenesis. Obeticholic acid, a BA derivative, is a dual agonist of these receptors, whose use in humans has shown a histological benefit in NASH patients in liver biopsies ${ }^{19}$. In LDL -/- mice, the activation of TGR 5 slowed the development of atheroma by targeting the macrophages in the plaque, decreasing the production of pro-inflammatory cytokines and inhibiting the oxidized LDL uptake by lowering the expression of $\mathrm{CD} 36^{8}$. A similar protective role has also been attributed to FXR. On a high fat diet, the plaque development is $50 \%$ greater in double KO LDL-/- and FXR -/-, than in simple KO LDL -/-mice, with similar effects observed in ApoE -/- mice ${ }^{20,21}$. Of note, atherosclerosis is more than a mere aging process, implying the participations of pro-inflammatory and metabolic disorders ${ }^{1}$ : several studies in animals and humans have linked the stimulation of BA receptors to other beneficial metabolic effects, in energy expenditure ${ }^{22}$ or glucido-lipid metabolism ${ }^{6}$.

In order to specifically address the question of the clinical relevance of CAD and BA in human, the main objective of our study was to compare the BA pool of patients with versus without angiographically confirmed coronary artery disease (CAD). The microbiota being a determining factor in the qualitative composition of the BA pool, the second objective was to compare the microbiota composition of the two groups discriminated by coronary angiograms. Applying rigorous inclusion criteria to prevent variations in the microbiota and $\mathrm{BA}$ metabolism ${ }^{23}$, we measured the serum and fecal BA concentrations and composition. We estimated the daily liver synthesis by measuring 7-alpha C4, a precursor molecule ${ }^{24}$, and the microbiota composition by 454 pyrosequencing.

\section{Results}

Study population. Between February and May 2015, 406 patients were hospitalized to undergo a scheduled coronary angiogram, of whom 326 were excluded for various criteria (Fig. 1). The indications for coronary angiogram and the disease severity are shown in supplementary table 2 . Ultimately, 80 patients were included in the study, of whom 45 presented with significant CAD and 35 were normal. The clinical characteristics of the two groups are detailed in Table 1. They were comparable except for 3 characteristics: age, gender and diastolic blood pressure. The group presenting with CAD was significantly older, more often men, and had a higher diastolic blood pressure.

Bile acids deficiency in sera is predictive of CAD. The total concentration of BA in sera (the sum of the 28 BA species) was two-fold lower in patients with than without CAD (Fig. 2A). By multiple variable analysis, 


\begin{tabular}{|l|l|l|l|}
\hline & NCAD $(N=35)$ & CAD $(N=45)$ & $P$ \\
\hline Demographic characteristics & & & \\
\hline Male gender & $17(49 \%)$ & $33(73 \%)$ & $P=0.02$ \\
\hline Age & $57 \pm 2.3$ & $66 \pm 1.3$ & $P=0.004$ \\
\hline Cardiovascular risk factor & & & \\
\hline Weight $(\mathrm{kg} 0$ & $74 \pm 2.8$ & $77 \pm 1.7$ & $P=0.28$ \\
\hline Height $(\mathrm{m})$ & $1.7 \pm 0.02$ & $1.72 \pm 0.01$ & $P=0.20$ \\
\hline BMI $\left(\mathrm{kg} / \mathrm{m}^{2}\right)$ & $26 \pm 0.75$ & $25.9 \pm 0.5$ & $P=0.64$ \\
\hline Hypertension & $10(29 \%)$ & $21(47 \%)$ & $P=0.11$ \\
\hline Systolic blood pressure $(\mathrm{mmHg})$ & $132 \pm 3.6$ & $138 \pm 3.7$ & $P=0.21$ \\
\hline Diastolic blood pressure $(\mathrm{mmHg})$ & $72 \pm 2.2$ & $77 \pm 1.8$ & $P=0.04$ \\
\hline Smoke & $16(46 \%)$ & $22(49 \%)$ & $P=0.82$ \\
\hline Dyslipidemia & $1(3 \%)$ & $5(11 \%)$ & $P=0.22$ \\
\hline Diabetes & $6(17 \%)$ & $8(18 \%)$ & $P=1$ \\
\hline Hereditary & $6(17 \%)$ & $9(20 \%)$ & $P=0.78$ \\
\hline Biological results & & & \\
\hline Total cholesterol $(\mathrm{g} / \mathrm{l})$ & $2 \pm 0.17$ & $2 \pm 0.17$ & $P=0.42$ \\
\hline Triglycerides $(\mathrm{g} / \mathrm{l})$ & $1.2 \pm 0.1$ & $1.3 \pm 0.11$ & $P=0.09$ \\
\hline HDL $(\mathrm{g} / \mathrm{l})$ & $0.6 \pm 0.05$ & $0.6 \pm 0.05$ & $P=0.36$ \\
\hline LDL $(\mathrm{g} / \mathrm{l})$ & $0.9 \pm 0.09$ & $1.15 \pm 0.10$ & $P=0.53$ \\
\hline Creatinine level $(\mu \mathrm{mol} / \mathrm{l})$ & $85 \pm 10$ & $81 \pm 3.2$ & $P=0.17$ \\
\hline
\end{tabular}

Table 1. Clinical characteristics of patients with and without coronary artery disease. NCAD: No Coronary Artery Disease. CAD: Coronary Artery Disease.
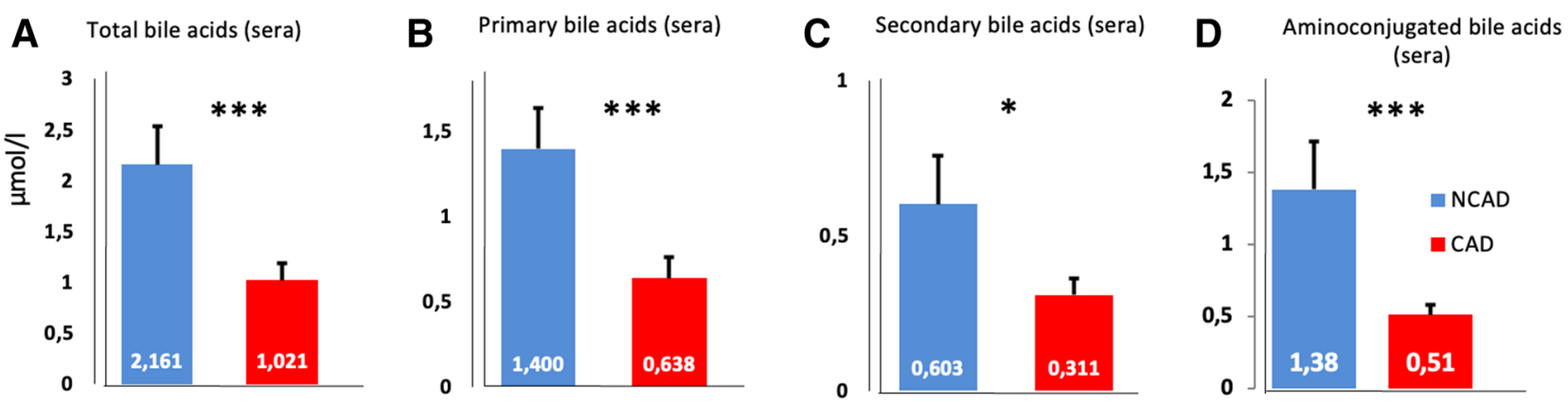

Figure 2. Comparison of bile acids concentrations in sera in non-coronary artery disease (blue bars) and coronary artery disease (red bars) patients. Concentrations ( $\mu \mathrm{mol} / \mathrm{l})$ of total $(\mathbf{A})$, primary $(\mathbf{B})$, secondary $(\mathbf{C})$ and amino-conjugated (D) bile acids in sera. Image credit: Graphpad 6.0, https://www.graphpad.com/scientificsoftware/prism/.

the total BA concentration, adjusted for age and gender, was highly predictive of CAD. The area under the curve (AUC) was $83.6 \%(74.5-92.8 \%)$, and OR $(95 \% \mathrm{CI})$ was $0.51(0.31,0.85), P=0.01$ (Fig. $4 \mathrm{~A}$; supplementary table 3 ).

The primary, secondary, and conjugated serum BA concentrations were decreased and systematically lower in patients with, than in patients without CAD (Figs. 2B-D). However, the proportions of primary, secondary or conjugated BA were similar, with a global decrease in BA species in patients presenting with CAD (supplementary table $4 \mathrm{~B}$ ).

We therefore sought to identify a candidate bile acid that could be a single marker reflecting the overall decline in the 28 circulating bile acid species. We oriented our analysis towards G-CDA, described in the literature as the primary bile acid synthesized most abundantly by the liver in comparison with other primary bile acids. Among the 28 BA isolated in sera, the glyco-conjugated GCDCA acid was the most abundant, representing $30 \%$ of all BA species, (supplementary figure 2). This proportion was similar in both groups (data not shown). Like total $\mathrm{BA}$, the concentration of GCDCA was twofold lower in patients with than in patients without CAD. The serum GCDCA concentration was similarly predictive of CAD, with an AUC of 83.6\% (74.1-93.0\%) and OR of 0.06 95\% CI 0.01, 0.51; $P=0.01$ (Fig. 4B; supplementary table 3 ).

Unchanged concentrations and composition of BA in feces of patients presenting with CAD. In contrast to the sera, the total concentrations of BA in feces samples were similar in both study sam- 

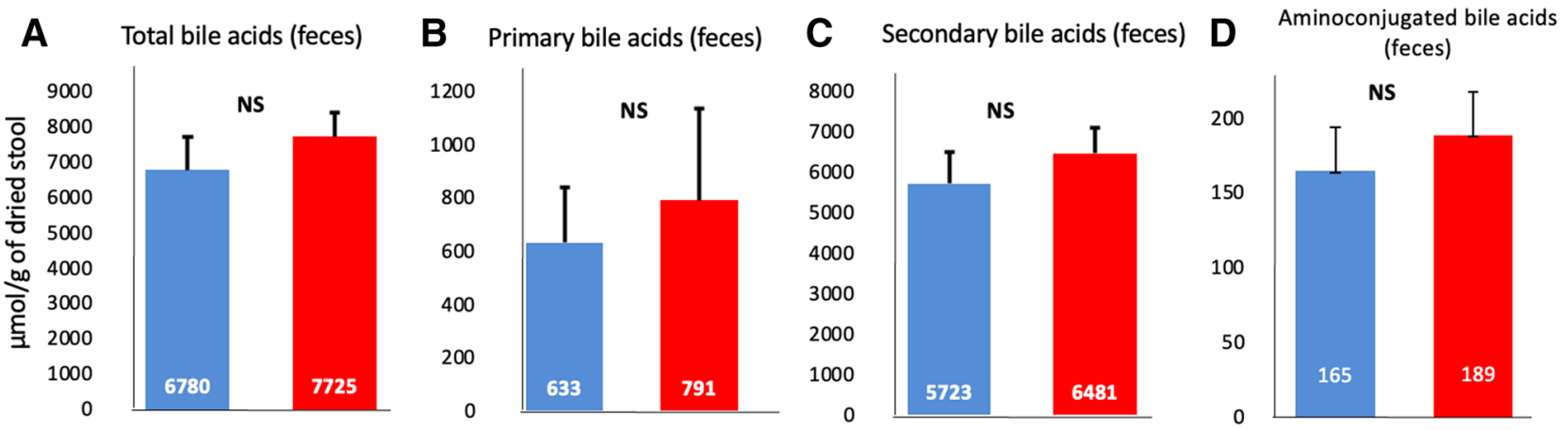

Figure 3. Comparison of bile acids concentrations in feces in non-coronary artery disease (blue bars) and coronary artery disease (red bars) patients. Concentrations ( $\mu \mathrm{mol} / \mathrm{g}$ of dried stools) of total (A), primary (B), secondary (C) and amino-conjugated (D) bile acids in feces. $P<0.05 ;{ }^{\star *} P<0.01 ;{ }^{\star * \star} P<0.001$. Image credit : Graphpad 6.0, https://www.graphpad.com/scientific-software/prism/.
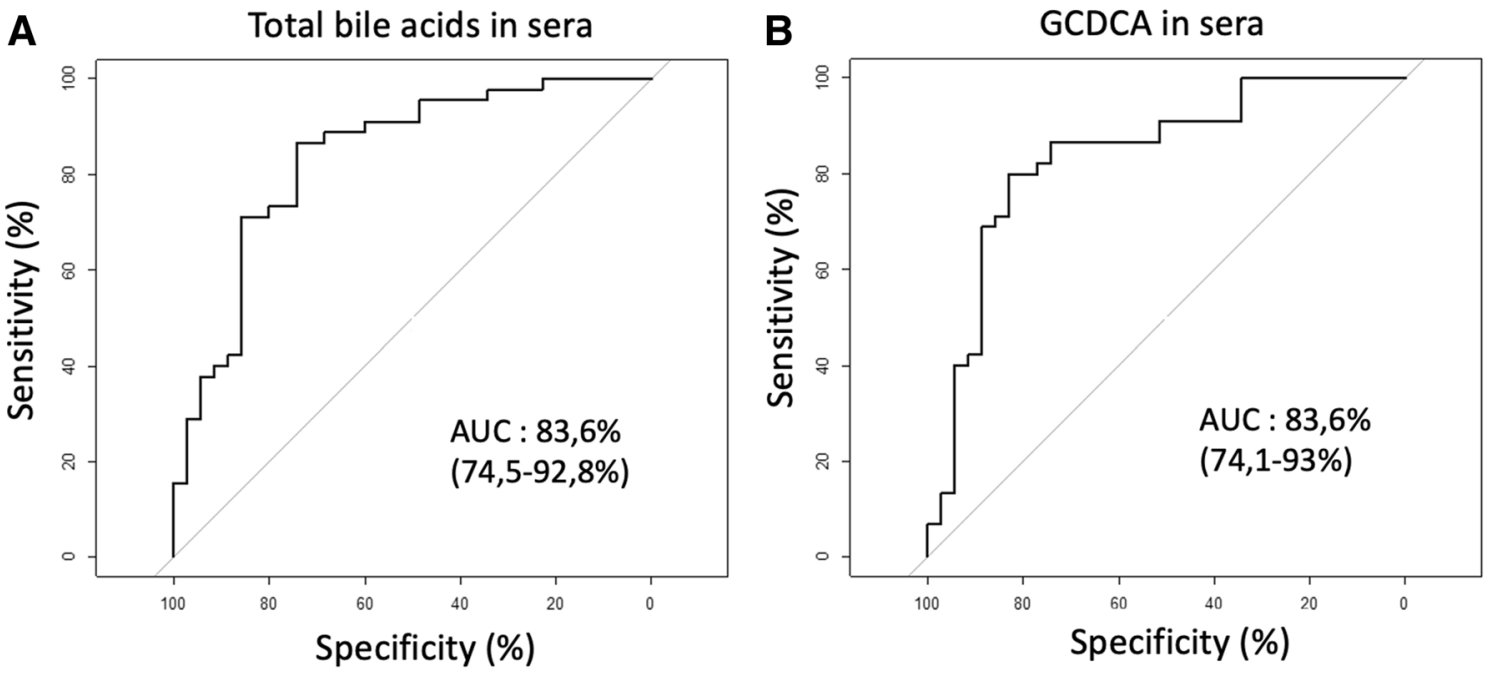

Figure 4. Receiver operating characteristic curves using as predictive factors of coronary artery disease adjusted for age and gender: (A) total bile acids concentrations in sera; (B) glycho-chenodeoxycholic acid (GCDCA) in sera. Image credit: R software: https://www.r-project.org.

ples (Fig. 3A). The main, primary, secondary, and conjugated species were also similar, in concentrations as well as proportions (Figs. 3B-D, and supplementary table 5).

Similar hepatic bile acids synthesis in patients with and without CAD. The measurement of 7alpha C4 in serum is an indirect reflection of the total daily hepatic synthesis of $\mathrm{BA}^{24}$. This metabolite is the first step of hepatic production of bile acids from cholesterol. It's produced by CYP7A1 enzyme, which is the rate-limiting enzyme in bile acid synthesis, and whose enzymatic activity measured on liver biopsy is directly correlated to the circulating levels of $7 a$-hydroxy-4-cholesten-3-one ${ }^{25}$. The concentrations of 7alpha C4 in sera was similar in the two groups $(0.13 \pm 0.01$ vs $0.12 \pm 0.01 \mu \mathrm{mol} / \mathrm{l}$; Figs. $4,5 \mathrm{~A})$.

Significant increase in the concentrations of bile acids by statins in the serum of patients with CAD. In the subset of 17 patients with CAD, the mean concentration of total BA doubled after 1 month of statin therapy, from $0.68 \pm 0.08$ to $1.37 \pm 0.21 \mu \mathrm{mol} / \mathrm{l}, P=0.01$; Fig. $5 \mathrm{~B})$. Total BA concentration increased in 13 of 17 patients (77\%) and decreased in 4 of 17 patients $(23 \%)$.

The supplementary figure 3 shows the individual evolution of total serum bile acid concentrations after one month in each of the 17 patients.

The fecal microbiota composition is not predictive or specific of CAD. The richness of fecal microbiota was similar in both groups. The overall number of species observed in a sample, represented by the alpha diversity (Fig. 6A) and other alpha diversity, represented with the Shannon, Simpson, and Chao-1 indexes (supplementary figure 1) were similar. The beta (inter-sample) diversity, represented by the Bray Curtis index, was also unspecific in patients with versus without CAD (Fig. 6B). While no bacterial clusters in the microbiota 
A

\section{7 alpha $\mathrm{C} 4$ concentration in sera}

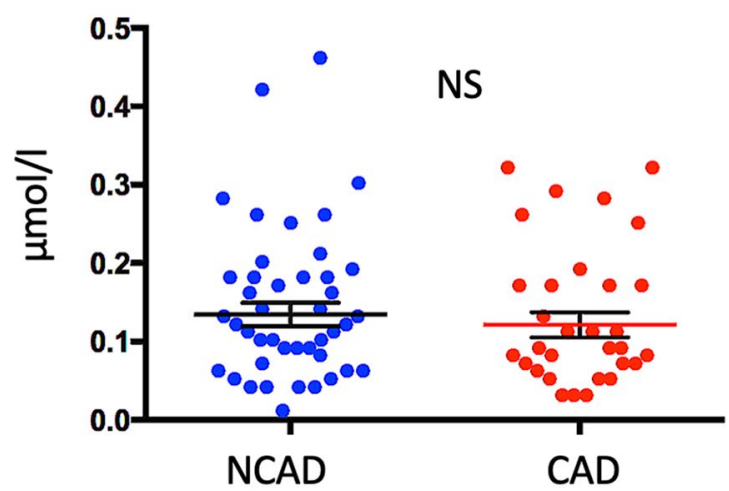

B

B

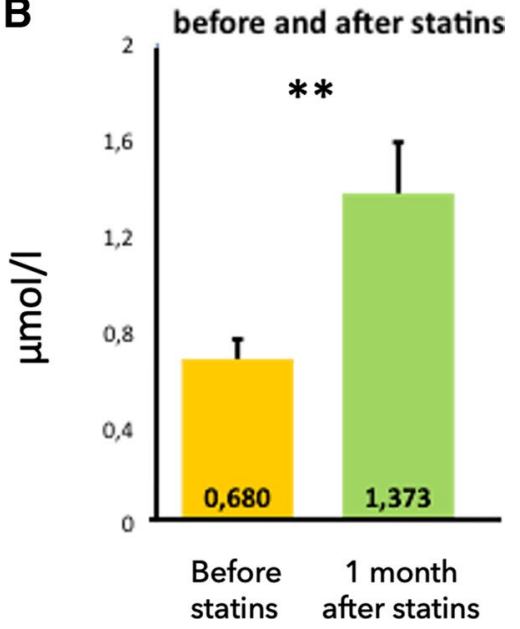

Figure 5. (A) Concentrations ( $\mu \mathrm{mol} / \mathrm{l}$ ) of 7 alpha $\mathrm{C} 4$, a liver metabolite reflecting the daily hepatic bile acid synthesis in sera in the two study samples. (B) Comparison of total bile acids concentrations in a subset of 17 patients with coronary artery disease after 1 month of statin therapy. NCAD = non-coronary artery disease; $\mathrm{CAD}:=$ coronary artery disease. ${ }^{*} P<0.01$. Image credit: Graphpad 6.0, https://www.graphpad.com/scientificsoftware/prism/.

composition was identified in any of the groups, the firmicutes phylum at the taxa levels were more prominently represented in the feces of patients with CAD (Fig. 6C).

\section{Discussion}

The objective of this work was to determine in humans whether there are observable qualitative or quantitative differences in circulating and fecal bile acids, and in the composition of the microbiota during CAD. We used strictly controlled sampling conditions in a carefully recruited population, justifying the $80 \%$ of non-inclusion of eligible patients, to avoid artificial variations induced by multiple factors that may influence the determination of circulating bile acids ${ }^{26}$. In a comparison of patients with and without CAD, we observed in patient with $\mathrm{CAD}$ a quantitative defect affecting all species of circulating bile acids, with an overall concentration halved. We identified GCDCA, a unique BA among the 28 acids, as a marker of this defect, and a predictive factor of CAD. We also observed that this decrease in BA could be corrected by the administration of statins. We observed no specific pattern nor in the qualitative repartitions of BA pool, neither composition of the fecal microbiota.

This work has some limitations: the first one is the identification of patients with versus without atheroma based on coronary angiograms. Since it reveals the presence of intraluminal arterial irregularities, coronary angiograms, as opposed to computed tomography scanning, may miss extra luminal atheromas. We chose coronary angiograms when designing the study, as our patients would not have systematically undergone scanning, whereas they all had a scheduled angiogram before their inclusion. Second, patients with CAD were older than patients without, as if it was not a surprising observation-aging being a risk factor for cardiovascular events. Moreover, our selective screening aimed at eliminating confounding factors : we can then consider that a weakness of the study is that NASH / NAFLD pathologies were not screened in the patient population, by non-invasive tests (liver elastometry combined with blood tests) ${ }^{27}$. Abnormalities of bile acid metabolism have been described in these metabolic pathologies ${ }^{28}$, which are associated with the development of atheroma. However, there was no difference between the two groups in terms of the associated co-morbidities that prompt the search of a NAFLD (obesity, metabolic syndrome, diabetes) ${ }^{29}$ and patients with alteration of liver enzymes were systematically excluded.

These observations support the hypothesis that BA could acts as natural braking mechanism, protecting against the development of atheromatous plaques. So far, in human the literature is scarce. Recently, Li et al. ${ }^{30}$ observed similar results in a cohort of more than 7400 patients, associating a decrease in circulating total bile acids with the presence, and also the severity, of CAD. Although the exclusion criteria did not include statin use, and although the enzymatic method used to measure bile acids is less accurate than HPLC MS/MS, it is interesting to note that the decrease in circulating bile acids could be observed in two prospective studies of large and small effective, that methodologically segregated two populations on coronary angiography data.

Our study does not provide any mechanistic approach for this observation. Through the literature, two different mechanistic axes supporting an anti-atherogenic effect of bile acids are proposed. Firstly, a molecular action of bile acids by their TGR 5 and FXR receptors, driving their anti-inflammatory effect in vitro ${ }^{31,32}$, as well as several beneficial actions on the metabolism in vivo ${ }^{6}$. Recent evidences that BA acts as hormones supports this molecular action, targeting their receptors through the serum with metabolic effect in various tissues ${ }^{33}$. The experimental observation of protection against atheroma comes mainly from the literature in animal models $\mathrm{s}^{8,20,21,34,35}$. Drugs targeting the BA receptors, such as obeticholic acid, had also promising effects in NAFLD, that is clinically associated to CVD ${ }^{19,36}$. But to date, no effect of a bile acid receptor-targeting drug is described in CAD. 


\section{A Alpha-diversity}

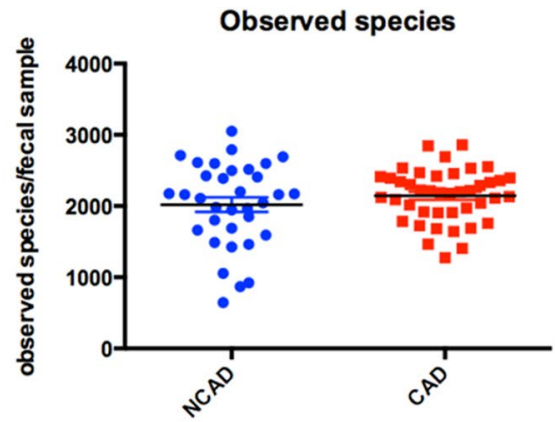

B Beta-diversity

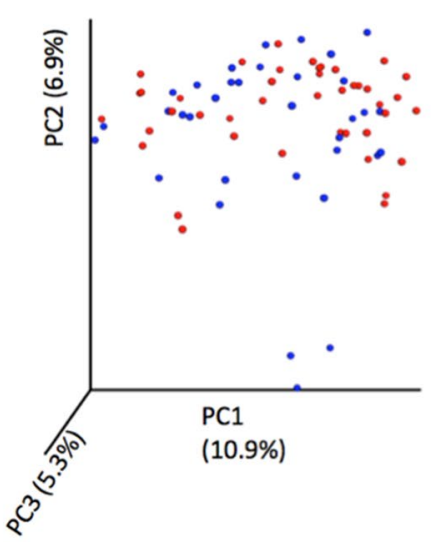

C

\section{Taxa enrichement comparison}

CAD $n=34$

NCAD $n=42$

Figure 6. Descriptive and comparative analysis of the fecal microbiota in both patient samples: (A) Intra sample bacterial diversity, represented by the alpha diversity index, and illustrated by the number of bacterial species observed in each fecal sample. (B) Inter sample bacterial diversity, represented by the beta diversity index, illustrated by the Bray Curtis distance analyzed by primary component analysis. (C) Bacterial taxa enrichment in patients with coronary artery disease using LeFSE analysis. * Taxa significant after adjustment for age and gender, using a multiple variable association with linear models. NCAD = Non-coronary artery disease; $\mathrm{CAD}=$ Coronary artery disease. Image credit: Figure A and (B): Graphpad 6.0, https://www.graphpad.com/scien tific-software/prism/. Figure (C): LEfsE, https://huttenhower.sph.harvard.edu/lefse/.

Secondly, the protective effect could be linked to a quantitative evacuation of BA, through their fecal excretion. The induction of an intestinal loss of BA after binding to BA sequestrants (colesevelam or cholestyramin) is a known pathway of cholesterol elimination through the gut, that can lower the concentration of LDL. ${ }^{37,38}$ Decreasing the BA pool, triggers a reaction in the liver, that promotes the conversion of the intracellular cholesterol to BA synthesis: colesevelam and cholestyramine could increase the circulating concentration of cholic acid, whereas the total BA concentrations remain stable or decrease ${ }^{39,40}$. Despite encouraging studies, the ability of bile acids sequestrants to slow the progression of CAD remains uncertain ${ }^{41}$.

Routinely prescribed after a cardiovascular event, lipid lowering statins therapy reduces in morbidity and mortality in $\mathrm{CVD}^{42}$. It also have been proven to be safe and possibly effective in the treatment of NAFLD/NASH ${ }^{43-45}$. Interestingly, we also observed that 1 month of statin therapy doubled the concentrations of circulating BA in patients with CAD. This subset of 17 patients began statin therapy for secondary prevention of CAD (16 patients received 10 to $80 \mathrm{mg}$ and 11 patients received $40 \mathrm{mg}$ of atorvastatin daily). In mice, atorvastatin causes an over expression of CYP7A1, the liver enzyme responsible of BA synthesis, and changes the BA pool composition ${ }^{46}$, without, however, increasing the pool of circulating BA. It is noteworthy that 17 of these 17 patients also received antiplatelets agents, 13 received a beta-adrenergic blocker, and 13 received an angiotensin converting-enzyme inhibitor. The increase in total BA might also be theoretically attributed to these other drugs, even though unlike statins, they do not target the cholesterol metabolism.

We have not described the mechanism explaining this decrease in the concentrations of circulating BA. With regard to the similar concentrations of 7alpha $\mathrm{C} 4{ }^{24}$ in the sera, the lower concentrations of BA in patients suffering from $\mathrm{CAD}$ is probably not the consequence of a lower synthesis (this metabolite is an indirect marker of the daily hepatic synthesis of $\mathrm{BA}^{24,25}$ ). Similarly, the similar fecal BA concentrations suggest that the intestinal excretion is unchanged in both groups. However, a spot sample is insufficient to measure the true excretion of 
BA, which requires a 24 to $48 \mathrm{~h}$ stool collection. Other studies have confirmed that the 24 -h intestinal excretion of $\mathrm{BA}$ is lower in patients with than in patients without $\mathrm{CAD}^{47}$. As if this lower excretion has been correlated with stroke and mortality ${ }^{48}$, these studies did no measure BA in serum or its synthesis, to support the hypothesis of low BA producer patients in CAD. We hypothesize a mechanism that causes variations in the systemic passage of BA allowed by the liver, from the enterohepatic to the general circulation. The supplementary Figure 4 summarize the main results of this work. It proposes a "spill over" mechanism of BA: in the same way of glycemic peak after the meal, this postprandial spillover of BA into the general circulation, has been proposed as an important metabolic factor to take into consideration ${ }^{16,49}$.

One of our initial hypotheses was that the microbiota unbalance observed in patients presenting with atheroma, might change the BA metabolism. We found neither changes in the microbiota diversity, nor in the BA composition or their fecal concentration. The variation at the taxa level we observed cannot be considered a specific or a mechanistic marker ${ }^{50}$. The composition of the microbiota is not a mean of evaluating its function, and it is clear that some metabolites of the intestinal microbiota, such as trimethylamine, a derivative of L-carnitine transformed in the liver to trimethylamine-N-oxide (TMAO), increase the risk of cardiovascular events ${ }^{51}$. The TMAO measurement could have been adressed in that work. Of note, administration of TMAO in mice alters bile acids profiles and lowers hepatic bile acids synthesis, by reducing Cyp7al enzyme expression ${ }^{52}$.

Compositional differences in the gut microbiota were frequently reported in patient with $\mathrm{CVD}^{53}$ while we have not documented any specific one in this work. Methodologic differences ${ }^{54}$ make comparison with our results is problematic. Differences in the composition of the microbiota have been reported in a comparison of 12 patients with symptomatic carotid atheromas and 13 controls. In addition to the anatomical location, the extent and size of pathological atheromatous plaques is also very different in peripheral atheroma (carotid or femoral) compared to coronary atheromatous, so patients may not be comparable. Another study, using the same pyrosequencing technique in 15 patients presenting with atherosclerotic disease and in 15 healthy subjects, found no differences in the composition of the fecal microbiota ${ }^{55}$.

We propose here GCDCA as a marker of global BA deficiency observed in CAD. This primary gluco-conjugated $\mathrm{BA}$ is the most abundantly synthetized by the liver in human ${ }^{26}$. We observed that its concentrations vary similarly in patients and in controls, and were similarly predictive of CAD than the total concentrations of BA (Fig. 4B and supplementary figure 2). This point is interesting because it could dispense with the determination of 28 bile acids to observe the decrease of bile acids in case of CAD, by dosing only the GCDCA in routine.

In conclusion, our study identified a decrease in the concentrations of circulating BA as a predictor of atheroma visible on coronary angiograms, in absence of difference in the fecal BA concentration or composition, or in the fecal microbiota diversity. This deficiency could be correctd by statins administration. Our observation in highly selected patients, confirms the results of other recent work in humans ${ }^{30}$, and points to the liver rather than to the gut, as the BA metabolism is tightly linked to the cholesterol metabolism (Supplementary figure 4). Regarding the experimental protective properties of BA, further studies are needed to decipher how this observation fit into the complex pathophysiological molecular mechanisms of coronary atherogenesis.

\section{Methods}

Patients. This study was conducted in accordance with the guidelines of the Helsinki declaration, defining the ETHICAL PRINCIPLES FOR MEDICAL RESEARCH INVOLVING HUMAN SUBJECTS, (2013). The work was reviewed and approved by an institutional ethic comitee: COMITE DE PROTECTION DES PERSONNES ILE DE FRANCE III (registration number S.C. 3218, 2015). All patients granted their written informed consent to participate. This work has been registered in ClinicalTrials and entitled "Bile Acids and Gut Microbiota as Potent Coronary Atheroma Risk Factors: a Prospective Study in Human (MABAC, ClinicalTrials.gov Identifier: NCT02375893).

All patients aged from 18 to 79 years old included between February and May 2015 underwent coronary angiograms. In the morning of angiogram, venous blood and sampling of feces were collected in the fasting state. After immediate centrifugation, the sera were frozen at $-80^{\circ} \mathrm{C}$ for later analysis. The fecal samples were immediately homogenized and frozen at $-80^{\circ} \mathrm{C}$. Patients in the non-fasting state or treated with statins or fibrates, corticosteroids, anti-human immunodeficiency virus agents, antimicrobials in the past 3 months, or suffering from liver disease or cancer, or who had experienced a recent cardiac arrest, or had undergone cholecystectomy, were excluded from this study.

Based on the coronary angiographic observations, the patients were divided between a group with or without coronary artery disease (CAD), regardless of its severity. In a subset of patients with CAD followed by our institution, we measured the quantitative effect of 1 month of statin therapy on BA in serum.

Bile acids measurements in blood and feces. BA were measured in sera and feces, as previously described $^{56}$, and detailed in the supplementary methods (supplementary table 1). Briefly, the BA were purified by solid phase extraction and measured, using a QTRAP ${ }^{\infty} 2000$, high-pressure liquid chromatography and tandem mass spectrometry system (Applied Biosystems/MDS SCIEX, Concord, Ontario, Canada). Each peak was identified by comparing the spectrum of a range including 28 species of BA. The data were acquired and measured using the Analyst V.1.4.2 software (AB-SCIEX). The BA measurements were expressed in concentrations or as the percentage of each specific BA $( \pm$ SEM) out of all BA after calibration of the method, with weighed mixtures and normalization relative to the internal standard (23-nor-5 $\beta$-cholanoic acid-3 $\alpha, 12 \alpha$-diol). For the analysis, the BA were grouped in categories (supplementary table 1) or expressed individually. To estimate the liver synthesis of BA, we measured the 7a-hydroxy-4-cholesten-3-one (7a-C4) concentrations in serum, the first specific metabolite produced by the liver during hepatic synthesis, the plasma concentration of which reflects the daily rate of bile acid synthesis by the liver in $\operatorname{man}^{24}$. The $\mathrm{C} 4$ extraction method was similar to the bile acids 
extraction and the $\mathrm{C} 4$ quantification was performed as previously described, using a deuterated $\mathrm{C} 4$ form as internal standard (7a-hydroxy-4-cholesten-3-one- $\mathrm{d} 7^{49}$.

Fecal microbiota and diversity analysis by 454 pyrosequencing method. The fecal bacterial diversity was studied as previously described ${ }^{57}$, and is detailed in the supplementary methods. Briefly, total fecal DNA was extracted. The bacterial diversity was determined in each sample by targeting part of the ribosomal genes. The bacterial species were identified by sequencing the 16sRNA gene V3-V4 hypervariable regions. Richness of the samples were summarized by standard alpha diversity indexes, representing the intra-diversity of each sample (number of bacterial species observed, Shannon, Simpson and Chaol index). The inter-sample diversity was represented by the Bray Curtis beta diversity index (principal component analysis of the Bray-Curtis distance). At the taxa classification level, the enrichment was compared using LEfSE analysis ${ }^{17}$, after adjustment for age and gender, using multivariate association with linear models.

Statistical analysis. A descriptive analysis of the clinical and laboratory characteristics of the patient population was performed. Quantitative data are expressed as means \pm standard deviation (SD) or median with interquartile range (IQR), and qualitative data as percentages with 95\% confidence intervals (CI). Correlations between clinical and laboratory data were examined, using statistical tests appropriate for the variables studied. Mean values were compared by Student's $t$-test or, if more appropriate, by Wilcoxon's test. Percentages were compared, using the chi-square test of Pearson 2, or by Fisher's exact test, if more appropriate. Logistic regression analyses were performed to examine the correlations between CAD and BA concentrations. Variables associated with CAD or its known risk factors, including hypertension, smoking, hyperlipidemia and diabetes, which emerged with a $<0.15$ degree of significance by single variable analysis, were entered in a multiple variable regression analysis. Odd ratios (OR) and their 95\% CI were calculated. All tests were two-sided with a 5\% significance level. The analyses were performed using the R software, https://www.r-project.org. From the statistical results obtained on the R software, some graphical figures were constructed using graphpad 6.0, https://www. graphpad.com/scientific-software/prism/. The specific analysis of the gut microbiota is detailed in the supplementary methods.

Received: 4 April 2021; Accepted: 9 November 2021

Published online: 22 November 2021

\section{References}

1. Bentzon, J. F., Otsuka, F., Virmani, R. \& Falk, E. Mechanisms of plaque formation and rupture. Circ. Res. 114, 1852-1866 (2014).

2. Stewart, J., Manmathan, G. \& Wilkinson, P. Primary prevention of cardiovascular disease: A review of contemporary guidance and literature. JRSM Cardiovasc. Dis. 6 (2017).

3. Stepanova, M. \& Younossi, Z. M. Independent association between nonalcoholic fatty liver disease and cardiovascular disease in the US population. Clin. Gastroenterol. Hepatol. Off. Clin. Pract. J. Am. Gastroenterol. Assoc. 10, 646-650 (2012).

4. Francque, S. M., van der Graaff, D. \& Kwanten, W. J. Non-alcoholic fatty liver disease and cardiovascular risk: Pathophysiological mechanisms and implications. J. Hepatol. 65, 425-443 (2016).

5. Santos, R. D., Valenti, L. \& Romeo, S. Does nonalcoholic fatty liver disease cause cardiovascular disease? Current knowledge and gaps. Atherosclerosis 282, 110-120 (2019).

6. Chávez-Talavera, O., Tailleux, A., Lefebvre, P. \& Staels, B. Bile acid control of metabolism and inflammation in obesity, type 2 diabetes, dyslipidemia and NAFLD. Gastroenterology https://doi.org/10.1053/j.gastro.2017.01.055 (2017).

7. Yu, Q., Jiang, Z. \& Zhang, L. Bile acid regulation: A novel therapeutic strategy in non-alcoholic fatty liver disease. Pharmacol. Ther. 190, 81-90 (2018).

8. Pols, T. W. H. et al. TGR5 Activation inhibits atherosclerosis by reducing macrophage inflammation and lipid loading. Cell Metab. 14, 747-757 (2011).

9. Kalhan, S. C. et al. Plasma metabolomic profile in nonalcoholic fatty liver disease. Metabolism 60, 404-413 (2011).

10. Yamamoto, S. et al. Bile acids aggravate nonalcoholic steatohepatitis and cardiovascular disease in SHRSP5/Dmcr rat model. Exp. Mol. Pathol. 114, 104437 (2020).

11. Uchida, K. et al. Altered bile acid metabolism related to atherosclerosis in alloxan diabetic rats. J. Atheroscler. Thromb. 3, 52-58 (1996).

12. de Aguiar Vallim, T. Q., Tarling, E. J. \& Edwards, P. A. Pleiotropic roles of bile acids in metabolism. Cell Metab. 17, 657-669 (2013).

13. Ridlon, J. M., Harris, S. C., Bhowmik, S., Kang, D.-J. \& Hylemon, P. B. Consequences of bile salt biotransformations by intestinal bacteria. Gut Microbes 7, 22-39 (2016).

14. Wang, H., Chen, J., Hollister, K., Sowers, L. C. \& Forman, B. M. Endogenous bile acids are ligands for the nuclear receptor FXR/ BAR. Mol. Cell 3, 543-553 (1999).

15. Maruyama, T. et al. Identification of membrane-type receptor for bile acids (M-BAR). Biochem. Biophys. Res. Commun. 298, 714-719 (2002).

16. Werling, M. et al. Enhanced fasting and post-prandial plasma bile acid responses after Roux-en-Y gastric bypass surgery. Scand. J. Gastroenterol. 48, 1257-1264 (2013).

17. Duboc, H., Taché, Y. \& Hofmann, A. F. The bile acid TGR5 membrane receptor: from basic research to clinical application. Dig. Liver Dis. Off. J. Ital. Soc. Gastroenterol. Ital. Assoc. Study Liver 46, 302-312 (2014).

18. Gonzalez, F. J., Jiang, C. \& Patterson, A. D. An intestinal microbiota-farnesoid X receptor axis modulates metabolic disease. Gastroenterology 151, 845-859 (2016).

19. Neuschwander-Tetri, B. A. et al. Farnesoid X nuclear receptor ligand obeticholic acid for non-cirrhotic, non-alcoholic steatohepatitis (FLINT): a multicentre, randomised, placebo-controlled trial. Lancet Lond. Engl. 385, 956-965 (2015).

20. Hanniman, E. A., Lambert, G., McCarthy, T. C. \& Sinal, C. J. Loss of functional farnesoid X receptor increases atherosclerotic lesions in apolipoprotein E-deficient mice. J. Lipid Res. 46, 2595-2604 (2005).

21. Hartman, H. B. et al. Activation of farnesoid X receptor prevents atherosclerotic lesion formation in LDLR-/- and apoE-/- mice. J. Lipid Res. 50, 1090-1100 (2009). 
22. Watanabe, M. et al. Bile acids induce energy expenditure by promoting intracellular thyroid hormone activation. Nature 439, 484-489 (2006).

23. Fiamoncini, J., Curi, R. \& Daniel, H. Metabolism of bile acids in the post-prandial state. Essays Biochem. 60, 409-418 (2016).

24. Axelson, M., Aly, A. \& Sjövall, J. Levels of 7 alpha-hydroxy-4-cholesten-3-one in plasma reflect rates of bile acid synthesis in man. FEBS Lett. 239, 324-328 (1988).

25. Axelson, M., Björkhem, I., Reihnér, E. \& Einarsson, K. The plasma level of 7 alpha-hydroxy-4-cholesten-3-one reflects the activity of hepatic cholesterol 7 alpha-hydroxylase in man. FEBS Lett. 284, 216-218 (1991).

26. Voronova, V. et al. A physiology-based model of bile acid distribution and metabolism under healthy and pathologic conditions in human beings. Cell. Mol. Gastroenterol. Hepatol. 10, 149-170 (2020).

27. Petta, S. et al. Serial combination of non-invasive tools improves the diagnostic accuracy of severe liver fibrosis in patients with NAFLD. Aliment. Pharmacol. Ther. 46, 617-627 (2017).

28. Bechmann, L. P. et al. Free fatty acids repress small heterodimer partner (SHP) activation and adiponectin counteracts bile acidinduced liver injury in superobese patients with nonalcoholic steatohepatitis. Hepatol. Baltim. Md 57, 1394-1406 (2013).

29. Leoni, S. et al. Current guidelines for the management of non-alcoholic fatty liver disease: A systematic review with comparative analysis. World J. Gastroenterol. 24, 3361-3373 (2018).

30. Li, W. et al. Fasting serum total bile acid level is associated with coronary artery disease, myocardial infarction and severity of coronary lesions. Atherosclerosis 292, 193-200 (2020).

31. Calmus, Y. et al. Differential effects of chenodeoxycholic and ursodeoxycholic acids on interleukin 1, interleukin 6 and tumor necrosis factor-alpha production by monocytes. Hepatol. Baltim. Md 16, 719-723 (1992).

32. Keitel, V., Donner, M., Winandy, S., Kubitz, R. \& Häussinger, D. Expression and function of the bile acid receptor TGR5 in Kupffer cells. Biochem. Biophys. Res. Commun. 372, 78-84 (2008).

33. McGlone, E. R. \& Bloom, S. R. Bile acids and the metabolic syndrome. Ann. Clin. Biochem. 56, 326-337 (2019).

34. Mencarelli, A., Renga, B., Distrutti, E. \& Fiorucci, S. Antiatherosclerotic effect of farnesoid X receptor. Am. J. Physiol. Heart Circ. Physiol. 296, H272-281 (2009).

35. Miyazaki-Anzai, S., Masuda, M., Levi, M., Keenan, A. L. \& Miyazaki, M. Dual activation of the bile acid nuclear receptor FXR and G-protein-coupled receptor TGR5 protects mice against atherosclerosis. PLoS ONE 9, e108270 (2014).

36. Mudaliar, S. et al. Efficacy and safety of the farnesoid X receptor agonist obeticholic acid in patients with type 2 diabetes and nonalcoholic fatty liver disease. Gastroenterology 145, 574-582.e1 (2013).

37. Shepherd, J. Mechanism of action of bile acid sequestrants and other lipid-lowering drugs. Cardiology 76(Suppl 1), 65-71 (1989).

38. Sonnett, T., Robinson, J., Milani, P. \& Campbell, R. K. Role of colesevelam in managing heterozygous familial hypercholesterolemia in adolescents and children. Adolesc. Health Med. Ther. 1, 53-60 (2010).

39. Expert panel on detection, evaluation, and treatment of high blood cholesterol in adults. Executive summary of the third report of the national cholesterol education program (NCEP) expert panel on detection, evaluation, and treatment of high blood cholesterol In Adults (Adult Treatment Panel III). JAMA 285, 2486-2497 (2001).

40. Garbutt, J. T. \& Kenney, T. J. Effect of cholestyramine on bile acid metabolism in normal man. J. Clin. Invest. 51, 2781-2789 (1972).

41. Ross, S. et al. Effect of bile acid sequestrants on the risk of cardiovascular events: a mendelian randomization analysis. Circ. Cardiovasc. Genet. 8, 618-627 (2015).

42. Almeida, S. O. \& Budoff, M. Effect of statins on atherosclerotic plaque. Trends Cardiovasc. Med. 29, 451-455 (2019).

43. Dongiovanni, P. et al. Statin use and non-alcoholic steatohepatitis in at risk individuals. J. Hepatol. 63, 705-712 (2015).

44. Blais, P., Lin, M., Kramer, J. R., El-Serag, H. B. \& Kanwal, F. Statins are underutilized in patients with nonalcoholic fatty liver disease and dyslipidemia. Dig. Dis. Sci. 61, 1714-1720 (2016)

45. Kargiotis, K. et al. Resolution of non-alcoholic steatohepatitis by rosuvastatin monotherapy in patients with metabolic syndrome. World J. Gastroenterol. 21, 7860-7868 (2015).

46. Fu, Z. D., Cui, J. Y. \& Klaassen, C. D. Atorvastatin induces bile acid-synthetic enzyme Cyp7a1 by suppressing FXR signaling in both liver and intestine in mice. J. Lipid Res. 55, 2576-2586 (2014).

47. Charach, G., Rabinovich, A., Argov, O., Weintraub, M. \& Rabinovich, P. The role of bile acid excretion in atherosclerotic coronary artery disease. Int. J. Vasc. Med. 2012 (2012).

48. Charach, G. et al. Reduced bile acid excretion is an independent risk factor for stroke and mortality: A prospective follow-up study. Atherosclerosis 293, 79-85 (2020).

49. Lamaziere, A. et al. Distinct postprandial bile acids responses to a high-calorie diet in men volunteers underscore metabolically healthy and unhealthy phenotypes. Nutrients 12, E3545 (2020).

50. Remely, M. et al. Gut microbiota of obese, type 2 diabetic individuals is enriched in Faecalibacterium prausnitzii, Akkermansia muciniphila and Peptostreptococcus anaerobius after weight loss. Endocr. Metab. Immune Disord. Drug Targets (2016).

51. Schiattarella, G. G. et al. Gut microbe-generated metabolite trimethylamine-N-oxide as cardiovascular risk biomarker: a systematic review and dose-response meta-analysis. Eur. Heart J. 38, 2948-2956 (2017).

52. Ding, L. et al. Trimethylamine-N-oxide (TMAO)-induced atherosclerosis is associated with bile acid metabolism. Lipids Health Dis. 17, 286 (2018).

53. Ascher, S. \& Reinhardt, C. The gut microbiota: An emerging risk factor for cardiovascular and cerebrovascular disease. Eur. J. Immunol. 48, 564-575 (2018).

54. Karlsson, F. H. et al. Symptomatic atherosclerosis is associated with an altered gut metagenome. Nat. Commun. 3, 1245 (2012).

55. Koren, O. et al. Human oral, gut, and plaque microbiota in patients with atherosclerosis. Proc. Natl. Acad. Sci. USA 108, 4592-4598 (2011).

56. Humbert, L. et al. Bile acid profiling in human biological samples: comparison of extraction procedures and application to normal and cholestatic patients. J. Chromatogr. B Analyt. Technol. Biomed. Life. Sci. 899, 135-145 (2012).

57. Morgan, X. C. et al. Dysfunction of the intestinal microbiome in inflammatory bowel disease and treatment. Genome Biol. 13, R79 (2012).

\section{Author contributions}

Dr H.D. and Dr C.C.-N. designed the study, submitted the project to local ethic committee, handled funding, collected clinical datas, collected the samples and wrote, edited and approved the paper. Dr D.R., Mrs L.H., Dr H.S., Pr B.C., Dr A.B., Dr M.L.G., P.P.S. achieved the Bile Acid measurement in blood and feces, and have edited and approved the paper. P.H.S. achieved the microbiota measurements and performed the specific statistical analysis, drafted, edited and approved the paper. Mrs A.B., Dr H.A., Dr N.B., P.J.-M.T. prepared submission to the local ethic committee and performed all the statistical analysis (except for gut microbiota), edited and approved the paper. Dr M.S., Dr K.W., P.O.V. and P.D.D. designed the study, recruited all the patients, gather informed consents and medical datas, edited and approved the paper. 


\section{Funding}

Financial support this work was supported by grants from the Fédération Française de Cardiologie, grants entitled "Bourse de Master 2" for Dr Caroline Chong-Nguyen support, and "Allocation de recherche" to finance biological analysis and material supplies, received by Dr Henri Duboc on the lab fundings.

\section{Competing interests}

Dr. Chong-Nguyen reports grants from Federation française de cardiologie, during the conduct of the study Pr Denis Duboc reports personal fees from Pfizer, personal fees from Takeda, personal fees from Wawe life science, personal fees from Amgen, personal fees from Novartis, outside the submitted work; Dr Dominique Rainteau has no conflict of interest to declare Pr Harry Sokol reports personal fees from Danone, grants from Biocodex, personal fees from MSD, personal fees from Takeda, personal fees from Abbvie, personal fees from Astellas, other from Enterome, other from Maat, personal fees from Novartis, personal fees from BMS, outside the submitted work. Mrs Lydie Humbert has no conflict of interest to declare Pr Philippe Seksik has no conflict of interest to declare Mrs Adèle Bellino has no conflict of interest to declare Dr Hendy Abdoul has no conflict of interest to declare Dr Naim Bouazza has no conflict of interest to declare Pr Jean Marc Treluyer has no conflict of interest to declare Dr Malikaa Saadi has no conflict of interest to declare Dr Karim Wahbi has no conflict of interest to declare Dr Heithem Soliman has no conflict of interest to declare Pr. Benoit Coffin reports personal fees from Mayoly Spindler, personal fees from Kyowa Kirin, personal fees from Ipsen, personal fees from Mundipharma, personal fees from Abbott, outside the submitted work Dr André Bado has no conflict of interest to declare Dr Maude Le Gall has no conflict of interest to declare Pr Olivier Varenne reports grants and personal fees from Boston Scientific, personal fees from Astra Zeneca, personal fees from Abbott Vascular, personal fees from Servier, outside the submitted work Dr. Henri Duboc reports grants from FEDERATION FRANCAISE DE CARDIOLOGIE, from null, during the conduct of the study; personal fees from BIOCODEX, grants from SNFGE, outside the submitted work.

\section{Additional information}

Supplementary Information The online version contains supplementary material available at https://doi.org/ 10.1038/s41598-021-02144-y.

Correspondence and requests for materials should be addressed to H.D.

Reprints and permissions information is available at www.nature.com/reprints.

Publisher's note Springer Nature remains neutral with regard to jurisdictional claims in published maps and institutional affiliations.

(c) (i) Open Access This article is licensed under a Creative Commons Attribution 4.0 International License, which permits use, sharing, adaptation, distribution and reproduction in any medium or format, as long as you give appropriate credit to the original author(s) and the source, provide a link to the Creative Commons licence, and indicate if changes were made. The images or other third party material in this article are included in the article's Creative Commons licence, unless indicated otherwise in a credit line to the material. If material is not included in the article's Creative Commons licence and your intended use is not permitted by statutory regulation or exceeds the permitted use, you will need to obtain permission directly from the copyright holder. To view a copy of this licence, visit http://creativecommons.org/licenses/by/4.0/.

(C) The Author(s) 2021 